\title{
Towards the New Generation of Surgical Meshes with 4D Response: Soft, Dynamic and Adaptable
}

Sonia Lanzalaco, * Pau Turon, Christine Weis, Christian Mata, Eulàlia Planas, Carlos Alemán, and Elaine Armelin*

Dr. Sonia Lanzalaco, Dr. Christian Mata, Dr. Eulàlia Planas, Prof. Carlos Alemán, Dr. Elaine Armelin

Departament of Chemical Engineering

Universitat Politècnica de Catalunya

C/ d'Eduard Maristany, 10-14, Building I

08019 - Barcelona (Spain)

E-mails: elaine.armelin@upc.edu, sonia.lanzalaco@upc.edu

Dr. Sonia Lanzalaco, Prof. Carlos Alemán, Dr. Elaine Armelin

Barcelona Research Centre for Multiscale Science and Engineering

Universitat Politècnica de Catalunya

C/ d'Eduard Maristany, 10-14, Building I

08019 - Barcelona (Spain)

Prof. Carlos Alemán

Institute for Bioengineering of Catalonia (IBEC)

The Barcelona Institute of Science and Technology,

C/ Baldiri Reixac 10-12

08028 - Barcelona (Spain)

Dr. Pau Turon, Dr. Christine Weis

Research and Development Centre

B. Braun Surgical, S.A.

Carretera de Terrassa 121

08191 - Rubí (Spain)

Keywords: polypropylene meshes, thermosensitive hydrogels, surgical implants, dynamic devices 


\begin{abstract}
Herein, a facile approach towards transforming a bidimensional (2D) polypropylene flexible mesh material into fourth dimension (4D) dynamic system has been presented. The versatile platform, composed by a substrate of knitted fibres of isotactic polypropylene (iPP) mesh and a coating of thermosensitive poly( $N$-isopropylacrylamide-co- $N, N^{\prime}$-methylene bis(acrylamide) (PNIPAAm-co-MBA) hydrogel, covalently bonded to the mesh surface, after cold-plasma surface treatment and radical polymerization, is intended to undergo variations in its geometry by reversible folding/unfolding behaviour. The study is the first to trace the tridimensional movement of a flat surgical mesh, intended to repair hernia defects, under temperature and humidity control. An infrared thermographic camera and an optical microscope were used to evaluate the macroscopic and microscopic structure stimulus response. The presence of the PP substrate and the distribution of the gel surrounding the PP threads, affected both the PNIPAAM gel expansion/contraction as well as the time of folding/unfolding response. Furthermore, PP$g$-PNIPAAm meshes showed an increase in the bursting strength of $\sim 16 \%$ with respect to the uncoated mesh, offering a strongest and adaptable system for its future implantation in human body. The findings reported offer unprecedented application possibilities in the biomedical field.
\end{abstract}




\section{Introduction}

Highly sophisticated advanced materials and unprecedented revolution on materials fabrication have been reported in the last decades. ${ }^{[1-3]}$ Particularly impressive is the development of 4D printing technology, ${ }^{[4]}$ in which the objects generated by a 3D printer are able to self-assemble after certain stimulus, and modify themselves in an autonomous way. ${ }^{[5,6]}$ However, the "fourth dimension-4D" concept is not new. It was introduced in 1788 by Joseph-Louis Lagrange, an Italian-French mathematician, and it was referred to a three-dimensional architecture and one temporal transformation. ${ }^{[7]}$ Nowadays, such a concept has been reinvented and extended to other areas, as for example the biomedical field, with the emerging 4D-printing technologies. ${ }^{[8-10]}$

Such a new variants of the 4D concept opened the door to the customized fabrication of a myriad of medical devices, like scaffolds (stents, valves); ${ }^{[3]}$ to develop artificial tissues, ${ }^{[11,12]}$ like bones, cartilage, and vascular vessels; and to print a variety of cells and organs, ${ }^{[10,13,14]}$ by employing either hard or soft matter. ${ }^{[15-19]}$ Streamlining the production process of programmable and adaptive biomedical materials is an affordable challenge when solid and flat devices, hard and soft structures, or multi-material structures are envisaged. ${ }^{[16,20,21]}$ However, if we look at the geometry of textile devices, such as meshes, wound dressings, sutures and others, there are technical limitations for the 3D printing manufacture and, consequently, for the establishment of the 4D concept.

Prompted by the relevance and the impact of the topic, the scientific community and the biomedical companies are looking for a new kind of meshes for hernia repair, able to self-adapt, with reduced inflammatory risks due to foreign body reaction. ${ }^{[22,23]}$ Different strategies have been proposed, from alterations of the mesh structure (pore size, shape, transition from a heavyweight to a lightweight meshes, knitting design of 
the fibres), ${ }^{[24]}$ to its modification by means of surface coatings. ${ }^{[25,26]}$ The last approach has gained an increased attention because it allows to achieve protection of the implant from degradation and mitigation of the foreign body reaction. Here, an innovative approach is proposed to use a sensing coating material, based on a thermosensitive hydrogel, to improve the adaptability of the textile device and to convert a surgical mesh in a smart self-evolving and self-fixating device. The current concept is supposed to help to overcome one of the main problems connected with the utilization of such materials, the post-operative chronic pain caused by the rigidity of the material.

Our technology is grounded in a well-stablished textile fabrication of mesh material from threads (1D) to the flat mesh geometry (2D) and, finally, after the application a hydrogel coating, to the smart 4D scale. In our previous work, the covalent bonding of poly $\left(N\right.$-isopropylacrylamide) (PNIPAAm), crosslinked with $\quad \mathrm{N}^{\prime} \mathrm{N}^{\prime}-$ methylenebis(acrylamide) (MBA), onto polypropylene (PP) mesh surface was elucidated and the mechanism of oxygen plasma activation was discussed. ${ }^{[27]}$. It was also necessary to approach the biocompatibility of live organisms with the new bilayer system. In this context, the best in vitro results with fibroblast (COS-1) and epithelial (MCF-7) cells were obtained with a lightweight mesh substrate modified with a porous iPP- $g$-PNIPAAm bilayer system, prepared via PNIPAAm grafting for $2 \mathrm{~h}$ and with the lowest crosslinker content. ${ }^{[28]}$ The cell attachment is desirable to prove the biocompatibility of the new device, whereas the phenomena of de-adhesion is very appealing for the final application of the mesh, in order to obtain a material able to avoid adherence to viscera.

Taking into account the success of the covalent bond among the inert PP fibres and the thermosensitive hydrogel, as well as, the biocompatibility of the whole system, the novelty of the present work relies on the study of the fourth-dimension (4D) behaviour 
of iPP- $g$-PNIPAAm system when subjected to cycles of increase/decrease temperature and by considering different mesh configurations and humidity conditions.

Therefore, an inactive unresponsive device is converted to a dynamic structure by applying two consecutive steps of material assembly and by controlling the deposition of the gel by one-side or two-sides on the PP mesh complex architecture.

For instance, in the field of long-term implants, like non-absorbable meshes for hernia repair, the fabrication method (and further modification processes), to obtain the desired mesh geometry, must preserve the flexibility of its structure and its resistance to stretching forces. Additional experiments to evaluate the mechanical integrity of the pristine mesh and the coated mesh material were also performed.

\section{Results and Discussions}

\subsection{Strategy for the preparation of dynamic polypropylene mesh materials}

The presence of PNIPAAm on top of PP meshes, with two-dimensional (2D) geometry (Figure S1, Supporting Information), allowed the system to dynamically interact with the local environment. The folding/rolling and unfolding/unrolling behaviour of the whole system can be easily tuned by arranging the thermosensitive hydrogel as two-sides (Figure 1a, bilayer system) or one-side (Figure 1b, monolayer system) around the PP substrate and by controlling the hydrogel amount and gel porosity (i.e. grafting time and swelling properties). Figures 1a-b show the preparation steps of the monolayer and bilayer platforms, respectively, reflecting that after the initial grafting reaction (Figures 1a1-b1), the materials are dried at $37^{\circ} \mathrm{C}$ in order to block the gel conformation in its contracted shape (Figures 1a2-b2). The preparation of the bilayer PP-g-PNIPAAm mesh was already explained in our previous works. ${ }^{[27,28]}$ However, in the case of the monolayer design, that was not reported before, the grafting reaction is 
preceded by a curl up of the untreated mesh around a glass bar support, allowing to obtain the desired shape (rolled) and avoiding the deposition of the gel on the inner side of the mesh (Figures 1b1-b2). The sample preparation was detailed in the section 4.2.

The actuation mechanism and the thermosensitivity of iPP-g-PNIPAAm were analysed by means of unfolding/unrolling experiments, which converted the gel from its initial contracted shape to a final expanded one (from a2 to a3 and from b2 to b3). Photographs of the real systems, the bilayer mesh grafted with PNIPAAm on two-sides of PP filaments, wet/opened and dry/folded can be visualized in Figures 1c1-c2, respectively; whereas the monolayer system, wet/rolled is reported in Figure 1c3. As is shown, the wet systems are optically transparent, thanks to the hydration of PNIPAAm chains, whereas the dried samples are white and opaque due to the removal of water and gel solidification. The experimental section describes details for the preparation of the monolayer and bilayer iPP- $g$-PNIPAAm platforms.

\subsection{Approach to the 4D behaviour of polypropylene meshes functionalized with thermosensitive hydrogel}

Briefly, bilayer and monolayer samples were previously dried at $37{ }^{\circ} \mathrm{C}$ in a folded (Figure 1a2) or rolled (Figure 1b2) shapes, in order to block PNIPAAm chains in their contracted conformation, and to observe the opening angles under thermal stimulus. The unfolding/unrolling measurements were carried out by immersion of the meshes in PBS aqueous solution (wet condition) and sequential digital photographs and optical micrographs, at different temperatures, were recorded.

For the stimulus response behaviour in the wet condition, the temperature ranged from 38.0-40.0 ${ }^{\circ} \mathrm{C}$ to $29.0^{\circ} \mathrm{C}$, by cooling steps. This election was not intended to simulate the mesh behaviour in contact with living tissue but rather to study and better visualize the 
hydrophilic/hydrophobic transitions of the PNIPAAm chains blocked in the PP substrate (responsible for the aperture of the mesh and the appearance of the 4D structure). The experiment setup is consistent with previous folding-unfolding experiments performed with PNIPAAm scaffolds, composed by hydrogel actuators only, i.e. without tough supports, and a physiological medium (phosphate buffer saline, PBS, solution). ${ }^{[29,30]}$ The unfolding/unrolling angles were measured by two different ways, considering the bilayer or the monolayer configurations. The unfolding angle $(\theta)$ was calculated taking into account an initial orientation of $\theta_{0}\left(20^{\circ}\right)$, starting by the bilayer iPP- $g$-PNIPAAm mesh completely or almost completely folded. Therefore, the $\theta$ values plotted in Figure 2 were calculated as follow:

$$
\theta=\theta_{\mathrm{f}}-\theta_{0}
$$

(Equation 1)

where $\theta_{\mathrm{f}}$ is the final angle at the time $t$ and $\theta_{0}$ is the initial orientation of the mesh. Hydrogel length variation was also measured according to Equation 2 (Experimental section) and results can be seen in Figure S2 (Supporting Information).

Figure 2 illustrates the progressive thermo-induced opening of the bilayer iPP- $g$ PNIPAAm mesh and the corresponding values of unfolding angle for PNIPAAm grafted for $1 \mathrm{~h}$ and $2 \mathrm{~h}$, while the thermo-dependent equilibrium swelling ratios (ESR, Table S1, Supporting Information) shown in Figure S3c (Supporting Information). When the actuator is placed in a water bath at $37.7^{\circ} \mathrm{C}$ (Figure 2a, 1), in its initial dried state, and the temperature is progressively decreased until $29.0{ }^{\circ} \mathrm{C}$ (Figure 2a, 2), the mesh starts to unfold. A stable unfolding angle of $27^{\circ}$ is reached at $33.5^{\circ} \mathrm{C}(2)$ for the bilayer system prepared with $1 \mathrm{~h}$ of reaction (Figure 2a), whereas $34^{\circ}$ is obtained at about $30.0{ }^{\circ} \mathrm{C}$ for a higher content of hydrogel onto iPP 2D geometry (Figure $2 \mathbf{b}$ and $\mathbf{S 2}$, Supporting Information). Consecutive steps of cooling and heating were applied on both meshes, showing a reversible motion of the iPP-g-PNIPAAm treated during $1 \mathrm{~h}$, with an 
unfolding angle varying from $0^{\circ}$ to $27^{\circ}$ the time response of each step being of $\sim 65$ seconds for this small angle variation (Figure 2b). At temperatures higher than $33.5^{\circ} \mathrm{C}$, the complete hydration of the PNIPAAm chains precludes the formation of further forces and the unfolding angle remains approximately constant.

The same approach has been used to evaluate the 4D response of PP meshes grafted with PNIPAAm gel during 2 h. The sequence of digital images showed in Figure 2c represents the progressive opening angles during the first cooling stage. Figure $2 d$ shows the resulting irreversible behaviour after applying consecutive steps and the final constant unfolding angle of $84^{\circ}$ (Figure S4, Supporting Information). The arrangement of the gel around the PP fibres, which is illustrated in the Figures S3a1-b1 (Supporting Information), plays a vital role in the reversible motion observed. The expansion and the shrinkage of the hard-soft bilayer are affected from the growth direction of PNIPAAm, leading to a loose of the reversibility when the chains are oriented in the direction parallel to the PP fibres (Figures S3a3-b3, Supporting Information). These results evidence that the macro pores of the PP meshes, with any geometry, must be free of gel for the reversibility and reproducibility of time-dependent deswelling and swelling properties in PBS solution. The thermosensitive response of both systems can be visualized in the supplementary movies (movies MV1 and MV2, for PP-g-PNIPAAm generated with 1 $\mathrm{h}$ and $2 \mathrm{~h}$ of graft polymerization, respectively).

For the sake of comparison, a representative SEM micrograph of PP-g-PNIPAAm grafted during $4 \mathrm{~h}$ is reported in Figure S3b3 (Supporting Information), showing a bilayer system where the PP fibres are totally covered by an excess of grafting and gel amount. Such platform is supposed to be unsuitable for mesh implants because it cannot ensure the growth of cells across the mesh that would reduce the graft integration in the 
tissue. ${ }^{[28]}$ The unfolding experiments for this system are reported in the ESI (Figure S4, Supporting Information).

\subsection{Comparison of 4D behaviour of polypropylene meshes functionalized with one layer and two layers of thermosensitive hydrogel}

Monolayer mesh implants manifested a more stable 4D response than the bilayer systems. Figure 3 reports the chemical and the morphological characterizations, as well as the actuator mechanism of the PP-g-PNIPAAm in the rolled configuration. Firstly, to confirm the one side grafting, a comparison between Raman spectra were carried out on both, inner and outer sides of iPP mesh fibres (Figure 3a). The bands of the PNIPAAm gel, which correspond to the C-C stretching at $840 \mathrm{~cm}^{-1}$, amide II at $1460 \mathrm{~cm}^{-1}$ (C-N with $\mathrm{N}-\mathrm{H}$ ) and amide $\mathrm{I}$ at $1658 \mathrm{~cm}^{-1}$ (predominantly $\mathrm{C}=\mathrm{O}$ ), were only detected on the outer side of the mesh, indicating the successful strategy to obtain the monolayer system. This was confirmed by SEM micrographs carried out on the cross-section of PP/hydrogel material (Figure 3b).

Finally, the temperature-dependent motion of the rolled meshes, treated during $2 \mathrm{~h}$ and $4 \mathrm{~h}$ of polymerization reaction, has been carried out and the results are reported in Figures 3c-e and S5 (Supporting Information), respectively. Samples were previously dried at $40.0^{\circ} \mathrm{C}$ in a rolled shape, as shown in the inset of the Figure 3a. The unrolling angle $(\varphi)$, which is represented Figure 3c, was determined taking into account an initial fully rolled configuration as $0^{\circ}$ and the angle by AOB points, as defined in Figure $3 \mathbf{d}$.

When the actuator is placed in a water bath at $40.0^{\circ} \mathrm{C}(\mathbf{1})$ in its initial dried state and the temperature is decreased till $29.0^{\circ} \mathrm{C}(2)$, the mesh starts to unroll until a stable angle of $40^{\circ}$ is reached at $30.0^{\circ} \mathrm{C}$. After this first opening occurred by decreasing the temperature, an additional unrolling movement is observed in the next heating step from 29.0 to $40.0{ }^{\circ} \mathrm{C}$. Digital 
photography corresponding to $40.0^{\circ} \mathrm{C}$ (4) shows the maximum bending angle reached $\left(85^{\circ}\right)$ (Figure 3e). Additional steps of cooling and heating did not generate further motions (Figure 3d), indicating that the 4D-response reached a stable non-cyclic movement. Unlikely, the samples belonging from $4 \mathrm{~h}$ of grafting has poor response of opening and closing angles, proving that high amount of hydrogel (high ESR, Table S1, Supporting Information) is not advantageous to get a thermosensitive device due to the over-crosslinked hydrogel chains blocking the PP threads.

In both, the bilayer (Figure 2) and in the monolayer (Figure 3) systems, the mesh starts to unfold/unroll due to the intramolecular forces applied on the PP filaments by the elongated PNIPAAm chains. This effect is associated with the creation of hydrogen bonds among polar groups (amides) inside PNIPAAm structure and the destruction of hydrogen bonds with water molecules from the PBS medium, causing the appearance of unfolding forces. ${ }^{[30-34]}$ Thus, the amount of water absorbed or desorbed by the hydrogel, which allows the expansion or the shrinkage of the PNIPAAm chains, respectively, is intrinsically related to the motion of the mesh (appearance of 4D response). Here, we prove that such behaviour is extremely dependent on the grafting reaction time of NIPAAm and on the swelling properties of the gel.

\subsection{Mechanical properties evaluation}

In order to confirm the dimensional stability and the integrity of the coated mesh, the mechanical properties should be evaluated and should match the conditions required by the biomechanical microenvironment of the human body. A crucial parameter is the intra-abdominal pressure (IAP), that could be responsible for adverse effects (i.e. decreased cardiac output, reduced hepatic perfusion, decreased renal blood flow), as well as for the formation and recurrence of hernias. ${ }^{[35]}$ Polypropylene mesh has been shown 
to have a bursting strength more than 10 times the maximum value predicted for the abdominal wall $(16 \mathrm{~N} / \mathrm{cm}) \cdot{ }^{[36]}$ In this sense, eventual modification produced by the plasma treatment and by the grafting of the hydrogel over fibres should also be evaluated. Figures 4a-c show digital images of the equipment and the meshes without and with gel submitted to the bursting test and the mechanical response of breaking strength and elongation values (Figure 4d), obtained on five studied samples for each kind of mesh. According to the results, comparing the PP mesh fibres untreated (U-PP), treated by $\mathrm{O}_{2}$-plasma (T-PP) and covered by the gel (PP- $g$-PNIPAAm), the presence of the hydrogel led to highest maximum values of strength and elongation at break.

Comparing the PP raw material (U-PP) and plasma treated sample (T-PP), the strength at break of the last has been slightly decreased $(241.8 \pm 25.0 \mathrm{~N}$ and $231.9 \pm 12.7$ $\mathrm{N}$, for U-PP and T-PP, respectively) as well as its deformation at break $(11.4 \pm 0.85 \mathrm{~mm}$ and $10.3 \pm 0.28 \mathrm{~mm}$, for U-PP and T-PP, respectively). According to Hwang et al. ${ }^{[37]}$ the mechanical properties of plasma treated polypropylene fabrics are sometimes affected by changes in roughness, that leads to an increased resistance, or by degradation that, oppositely, provoke a decrease. As we previously reported, ${ }^{[27]}$ the roughness of the mesh is not affected by the oxygen plasma treatment applied, thus, the subtle differences found on the mechanical properties cannot be ascribed to any degradation of the fibres surface. Conversely, the effect of PNIPAAm gel on the iPP fibres is more noticeable; either the strength $(267.3 \pm 18.6 \mathrm{~N})$ or the deformation at break $(12.0 \pm 0.60 \mathrm{~mm})$ have been increased if compared to the pristine samples (Figure 4d). Therefore, the presence of PNIPAAm hydrogel favours the mechanical anchorage of the mesh, allowing an interlocked structure developed upon the fibre surface and among fibre-to-fibre structures. 


\subsection{The macroscopic 4D behaviour of polypropylene meshes functionalized with thermosensitive hydrogel}

From a biomedical point of view, under physiological conditions, the new smart mesh material developed here would be able to detect local temperature changes in the zone where it will be implanted, thanks to the presence of the biocompatible PNIPAAm hydrogel. It is of outmost importance to evaluate the coated mesh behaviour outside human body, since the temperatures and atmosphere conditions will vary from its fabrication to its final utilization, passing by the sterilization processes as well. The synthetic implant will suffer changes from dry to wet (and vice-versa) as well as from lower to higher temperatures before mesh implantation, and must resist on flexibility and on bursting strength properties.

To follow-up the PP-g-PNIPAAm thermosensitive response of the biomedical platform on air, the macroscopic movement (4D response) was monitored by thermal infrared (IR) imaging, under high humidity condition (relative humidity of 100\%) and utilizing a PP mesh with size similar to those employed for final uses $\left(10 \times 10 \mathrm{~cm}^{2}\right)$.

Infrared (IR) imaging is one of the most powerful techniques based on infrared radiation. The signal produced by each sensor is proportional to the irradiation of heat and transformed into temperature, through emissivity and the distance of the object. Details on the experiment setup and infrared camera characteristics are supplied in the section 4.4.

As can be seen in the Figure 5, the unfolding angles are similar to those obtained in immersion solution. The sequence of images from left to right reveals an angle of $85^{\circ}$ when the mesh reaches the temperature of $38.0{ }^{\circ} \mathrm{C}$, upon heating process. The total opening time being 15 min. By the histogram analysis, the manifested higher pixel counts and temperature range 
are between $34.0-36.0{ }^{\circ} \mathrm{C}$, even if the chamber overpasses such temperatures. It confirms the stability of the new bilayer material in such temperature interval.

In conclusion, the absence of aqueous solution impacts on the time responsiveness of the whole system, by delaying the water desorption of the constrained PNIPAAm chains, but not on the thermosensitivity stability and cyclic behaviour of the bilayer platform. Comparison of the devices obtained after $2 \mathrm{~h}$ of polymerization reaction indicates that the platform evaluated on air has an unfolding angle of $80^{\circ}$ at $37.0{ }^{\circ} \mathrm{C}$ with a time response of $13 \mathrm{~min}$, whereas the immersed device takes $\sim 6$ min to unfold until $84^{\circ}$ at the same temperature. The fast heat transfer through the solution bath is the responsible for the marked decrease of self-unfolding time in the latter. The next research step will be focused on both the study of folding/unfolding response after sterilization of the mesh implant, a preliminary requisite to extend the research to in vivo pre-clinical studies, and to study the behaviour of the mesh using animal models to assess the response of the 4D mesh implant in contact with living tissue.

Up to now, there is no other studies on PNIPAAm based textiles fabricated, upon plasma activation, than those reported by our group. ${ }^{[27],[28]}$ Frequently, investigations are related to PNIPAAm derivatives for soft robotics $23,28,30-31$ and micro-actuator devices, ${ }^{[30,39,40]}$ the latter ones being composed of combinations of various soft hydrogels. Moreover, there have been few attempts towards in situ detection of the effect of temperature and humidity in the $4 \mathrm{D}$ response of thermosensitive materials. ${ }^{[41-45]}$

\section{Conclusion}

Here, a humidity- and temperature-sensitive mesh material has been created thanks to an easy strategy. A 2D knitted structure has been transformed into a dynamic and responsive system, after plasma activation and hydrogel grafting. The monolayer PP-g- 
PNIPAAm platform has been demonstrated to be highly promising smart material, with excellent dimensional stability to adapt to small bending type forces. The new system adopts a $4 \mathrm{D}$ scale that can be perfectly adaptable to soft tissue environments, as is the case of surrounding tissues in hernia repair surgery. The bursting strength and the elongation at break of the coated system were slightly improved with respect to the raw material, representing another advantage for the mesh adaptation to abdominal wall. Moreover, the samples are easily fabricated by a solid and reproducible method and either the dried or the wet meshes are handled without complications.

An important conclusion, beyond the results reported here, is that the $4 \mathrm{D}$ response achieved, for any of the tested configurations of PNIPAAm around PP threads, is not dependent on the hydrogel LCST temperature but rather governed by the distribution of PNIPAAm porous gel around the PP threads.

The actuator here fabricated presents advantages respect to commercial nonabsorbable mesh implants, consisting in the ability to self unfold thanks to the presence of the hydrogel coating. After mesh implantation by the surgeons (in vivo), the new system is expected to self-adapt to the abdominal wall during post operatory period, which is not possible with conventional coated meshes.

Taking into account that, up to now, the 3D-printing of PP mesh, by using 3D-CAD models, and the deposition of PNIPAAm hydrogel on each PP thread, by "printing" technology, are both unfeasible; the unique procedure to create a stable thermosensitive mesh material with 4D stimulus behaviour is that reported here. 


\section{Experimental section}

\subsection{Materials}

Optilene ${ }^{\circledR}$ Mesh LP, commercialized by B Braun Surgical S.A. (Rubí, Spain), was used as substrate for the polymerization reactions and deposition of the hydrogel. The surgical meshes are fabricated with non-absorbable isotactic polypropylene (iPP) yarns, manufactured by textile production, to convert the individual filaments to a monofilament mesh, lightweight $\left(36 \mathrm{~g} / \mathrm{cm}^{2}\right)$, with $1.0 \mathrm{~mm}$ of pore size and $0.39 \mathrm{~mm}$ of thickness. $N$-isopropylacrylamide (NIPAAm) was used as main monomer (purity 99\%, CAS 2210-25-5), $N, N^{\prime}$-methylene bis(acrylamide) (MBA) was used as co-monomer and crosslinker agent (Reagent Plus 99\%, CAS 110-26-9) and $N, N, N^{\prime}, N^{\prime}$-tetramethylethylenediamine (TEMED, Reagent Plus 99\%, CAS110-18-9) was employed as initiator. All chemical reagents were supplied by Sigma-Aldrich (Spain). Ammonium persulfate (APS, purity 98\% CAS7727-54-0) was employed as catalyst and was provided by Panreac S.A. (Spain). All reagents were used as received. Milli-Q water grade $(0.055 \mathrm{~S} / \mathrm{cm})$ and nitrogen gas in pure grade $(99.995 \%$ purity) were employed in all synthetic processes. For the PBS solution, Dulbecco's phosphate buffered saline (PBS) solution without calcium chloride and magnesium chloride were purchased from Sigma-Aldrich (USA) and prepared by dissolving $9.6 \mathrm{~g}$ of powder in $1 \mathrm{~L}$ of Milli-Q water.

\subsection{Procedure to prepare the monolayer platforms composed by iPP mesh material and PNIPAAm thermosensitive hydrogel}

For the preparation of the monolayer PP- $g$-PNIPAAm platforms the procedure was similar to the bilayer system, with the blocking of PP mesh in a glass tube to hinder hydrogel grafting in both sides. More in details, the PP mesh, previously functionalized with $\mathrm{O}_{2}$-plasma as described before, ${ }^{[27]}$ was cut into $2 \times 2 \mathrm{~cm}$ pieces, weighted and rolled on a glass tube; the mesh was laterally fixed by means of a PTFE tape and immersed in the polymerization solution. 
Grafting reactions of NIPAAm $(250 \mathrm{mM})$ in the presence of MBA as crosslinker $(1 \mathrm{mM})$, APS $(2.77 \mathrm{mM})$ and TEMED $(2.77 \mathrm{mM})$, as catalyst and radical initiator, respectively; were carried out in water solution for different times ( $1 \mathrm{~h}, 2 \mathrm{~h}$ and $4 \mathrm{~h})$. Afterwards, the samples were washed in distilled water, dried under vacuum and weighted to evaluate the graft yield.

The characterization of the monolayer and bilayer surgical meshes, as well as the grafting yield (GY) and equilibrium swelling ratio (ESR) were included in the supporting information.

\subsection{The unfolding/unrolling measurements to evaluate the thermosensitive motion of iPP- $g$-PNIPAAm platforms immersed in physiologic solution}

The actuation mechanism (4D response) and the thermosensitivity of the iPP-g-PNIPAAm were analysed by means of unfolding/unrolling experiments, which convert the gel from its initial contracted shape to a final expanded (Figure 1). The study was carried out with iPP-gPNIPAAm samples size of $2 \times 2 \mathrm{~cm}^{2}$ for the experiments carried out in solution and $10 \times 10 \mathrm{~cm}^{2}$ for the test carried out in air previously dried at $37{ }^{\circ} \mathrm{C}$ or $40{ }^{\circ} \mathrm{C}$ in an oven, to achieve a fully folded/rolled shape, in order to block PNIPAAm chains in their contracted conformation. Then, the folded mesh was immersed in a PBS bath (physiologic solution chose due to the biomedical application of the whole system) or in air with controlled humidity and the temperature was regulated among $29-40{ }^{\circ} \mathrm{C}$, with a heating controller. For the folding and unfolding angle measurements (where $\theta=0$ corresponds to the folded angle), a sequential digital photographs at different temperatures were recorded with a Nikon DX camera for the macroscopic images.

For the bilayer system (Figure 1, c1-c2), i.e. the PP mesh sandwiched between two layers of hydrogel, the unfolding angles was measured respect to the vertical line reported in the Figure 2c $(\theta=0$ corresponding to the folded angle for grafted and dried PP-g-PNIPAAm samples), whereas for the rolled samples (Figure 1, c3), the unrolling angle was measured by 
the opening representation showed in Figure 3d, both with decreasing temperature (from 40.0$37.0^{\circ} \mathrm{C}$ to $\left.29.0^{\circ} \mathrm{C}\right)$.

Additionally, the variation of the hydrogel length and the lower critical solution temperature (LCST), used to prove the thermoresponsiveness behaviour of the grafted hydrogel onto Optilene ${ }^{\circledR}$ Mesh LP surface, have been evaluated by optical microscopy by changing the temperature interval with a heating-cooling accessory. A Zeiss Axioscop 40 Pollight polarizing microscope (CarlZeiss, Göttingen, Germany) was employed to measure the expansion of PNIPAAm gel, while the temperature was controlled with a Linkam system having a THMS 600 heating and freezing stage connected to an LNP94 liquid nitrogen cooling system (LinkamScientific, Tadworth,UK). A Zeiss Axios Cam MRC5 digital camera (Carl Zeiss, Göttingen, Germany) was employed to get micrographs at appropriate temperature intervals. Such micrographs were used to calculate the distance between two sides from iPP fibre where the gel is grafted on it. Therefore, the variation of the gel length was evaluated according to the Equation 2:

Variation of hydrogel length $(\%)=\frac{L_{f}-L_{0}}{L_{0}} \cdot 100$

(Equation 2)

where $\mathrm{L}_{\mathrm{f}}$ and $\mathrm{L}_{0}$ are the lengths $(\mu \mathrm{m})$ of the PNIPAAm/MBA gel, well adhered to the mesh fibres, measured at the specific temperature and at the initial state, respectively.

\subsection{The unfolding measurements to evaluate the macroscopic 4D response of iPP-g- PNIPAAm platforms suspended on air}

The motion in air with controlled humidity conditions was carried out on a bilayer system with sizes similar to the ones implanted in the human body $\left(10 \times 10 \mathrm{~cm}^{2}\right)$ grafted during $2 \mathrm{~h}$, washed in water and dried overnight in the folded conformation and at temperature higher than the LCST to ensure the contraction of the PNIPAAm chains $\left(40^{\circ} \mathrm{C}\right.$ in an oven). 
After setting-up the system in the controlled humidity chamber, an infrared (IR) imaging camera was installed at $23 \mathrm{~cm}$ distance from the chamber and sequential images were taken. The IR camera used was the Thermal Imager Optris ${ }^{\circledR}$ PI connect 640 . This camera works on the spectral range wavelength of 7.5 to $13 \mathrm{~mm}$. A Focal Plane Array (FPA) is supported extracting a set of images with a definition of $640 \times 480$ pixels, the Optris PI 640 delivers pinsharp radiometric pictures and videos in real time. Moreover, the field of view (FOV) is $60^{\circ}$ in horizontal and $45^{\circ}$ in vertical respectively. This camera possesses a lens of $24^{\circ}$, and can measure temperatures ranging from $-20{ }^{\circ} \mathrm{C}$ to $900{ }^{\circ} \mathrm{C}$, by selecting diverse specific operating temperature ranges. It works as a radiometer composed by a set of bidirectional sensors. The signal produced by each sensor is proportional to the radiative heat emitted by the observed object. The signal is then transformed into temperature by means of the Stefan-Boltzmann equation, which requires the emissivity and the distance to the object. The operation mode is usually continuous, recording up to $32 \mathrm{fps}$ with a thermal sensitivity of less than $0.075{ }^{\circ} \mathrm{C}$. For this work, the operating temperature range used was from $0{ }^{\circ} \mathrm{C}$ to $150{ }^{\circ} \mathrm{C}$.

Two different softwares (ThermaCAM Researcher® and Matlab 2017b ®) were used for the image processing. Additionally, specifically ad-hoc algorithms were developed to obtain the set of histograms showed in Figure 5.

\subsection{Mechanical properties evaluation}

The bursting strength test, which is related to the performance determination of the maximum break strain of the meshes under compression forces, was carried out according to ASTM method D-3786, ${ }^{[46]}$ and by using a Zwick equipment (Model Z005) with $5 \mathrm{kN}$ load cell at a constant rate of $50 \mathrm{~mm} / \mathrm{min}$ and a distance between pendulum and the material of $0 \mathrm{~mm}$. Images of the equipment used and configuration of samples are reported in Figures 4a-c. Bilayer 
systems grafted during $2 \mathrm{~h}$ were selected to carry out the mechanical experiments. The samples were cut to $80 \times 80 \mathrm{~mm}^{2}$, and five specimens were tested for each condition: mesh untreated (U-PP) and treated by plasma (T-PP), and mesh coated by PNIPAAm gel (PP-g-PNIPAAm). Breaking strength (N) and elongation at break (\%) were measured to evaluate the alterations in the mechanical properties of the abovementioned samples. The meshes were previously wet by immersion in distilled water during $15 \mathrm{~min}$ at a temperature of $37^{\circ} \mathrm{C}$. After that, the samples were extracted and subjected to bursting test out of the solution, in order to evaluate the mechanical properties with the amount of water absorbed at desired conditions.

\section{Supporting information}

Supporting Information is available from the Wiley Online Library or from the authors.

\section{Acknowledgements}

This project has received funding from the European Union's Horizon 2020 research and innovation programme under the Marie Sklodowska-Curie grant agreement $\mathrm{N}^{\mathbf{o}}$ 796292 (Project 4D-POLYSENSE) and was also co-supported by MINECO (RTI2018098951-B-I00) and the Agència de Gestió d'Ajuts Universitaris i de Recerca (2017SGR359). B Braun Surgical S.A. (Spain) is also acknowledged for laboratory facilities and secondment supervision of Dr. Lanzalaco activities.

\section{Conflicts of interest}

The authors declare no conflict of interest.

\section{References}

[1] H. N. Chia, B. M. Wu, J. Biol. Eng. 2015, 9, 1.

[2] R. L. Truby, J. A. Lewis, Nature 2016, 540, 371.

[3] R. J. Morrison, S. J. Hollister, M. F. Niedner, M. G. Mahani, A. H. Park, D. K. Mehta, 
R. G. Ohye, G. E. Green, Sci. Transl. Med. 2015, 7, 1.

[4] S. Tibbits, Archit. Des. 2014, 84, 116.

[5] Q. Ge, C. K. Dunn, H. J. Qi, M. L. Dunn, Smart Mater. Struct. 2014, 23, 094007.

[6] F. Momeni, S. M.Mehdi Hassani.N, X. Liu, J. Ni, Mater. Des. 2017, 122, 42.

[7] J. L. Lagrange, Mécanique analytique; Cambridge University Press: Cambridge, 2009.

[8] Z. X. Khoo, J. E. M. Teoh, Y. Liu, C. K. Chua, S. Yang, J. An, K. F. Leong, W. Y. Yeong, Virtual Phys. Prototyp. 2015, 10, 103.

[9] J. An, C. K. Chua, V. Mironov, Int. J. Bioprinting 2016, 2, 3.

[10] B. Gao, Q. Yang, X. Zhao, G. Jin, Y. Ma, F. Xu, Trends Biotechnol. 2016, 34, 746.

[11] M. D. Sarker, S. Naghieh, N. K. Sharma, X. Chen, J. Pharm. Anal. 2018, 8, 277.

[12] J. Kobayashi, A. Kikuchi, T. Aoyagi, T. Okano, J. Biomed. Mater. Res. - Part A 2019, 107, 955.

[13] J. P. Jung, D. B. Bhuiyan, B. M. Ogle, Biomater. Res. 2016, $20,1$.

[14] L. Krauel, F. Fenollosa, L. Riaza, M. Pérez, X. Tarrado, A. Morales, J. Gomà, J. Mora, World J. Surg. 2016, 40, 889.

[15] S. E. Bakarich, R. Gorkin, M. In Het Panhuis, G. M. Spinks, Macromol. Rapid Commun. 2015, 36, 1211.

[16] T. van Manen, S. Janbaz, A. A. Zadpoor, Mater. Today 2018, 21, 144.

[17] T. Jungst, W. Smolan, K. Schacht, T. Scheibel, J. Groll, Chem. Rev. 2016, 116, 1496.

[18] M. Bodaghi, A. R. Damanpack, W. H. Liao, Smart Mater. Struct. 2016, 25, 105034.

[19] F. P. W. Melchels, M. A. N. Domingos, T. J. Klein, J. Malda, P. J. Bartolo, D. W. Hutmacher, Prog. Polym. Sci. 2012, 37, 1079.

[20] X. Meng, J. Hu, Z. Chao, Y. Liu, H. Ju, Q. Cheng, ACS Appl. Mater. Interfaces 2018, $10,1324$.

[21] L. Jing, K. Li, H. Yang, P. Y. Chen, Mater. Horizons 2020, 7, 54. 
[22] T. N. Robinson, J. H. Clarke, J. Schoen, M. D. Walsh, 2005, 1556.

[23] S. Kumar, R. G. Wilson, S. J. Nixon, I. M. C. Macintyre, Br. J. Surg. 2002, 1476.

[24] S. G, O. DR, P. H, J Urol 2012, 188, 27.

[25] P. J. Emans, M. A. R. C. H. F. SChreinemacher, M. A. J. J. G. Ijbels, G. E. L. B. Eets, J. A. N. I. M. G. Reve, L. E. O. H. K. Oole, N. I. D. B. Ouvy, Ann. Biomed. Eng. 2009, $37,410$.

[26] M. Bredikhin, D. Gill, J. Rex, W. Cobb, R. A, V. Vertegel, Hernia 2020.

[27] S. Lanzalaco, P. Turon, C. Weis, C. Alemán, E. Armelin, Soft Matter 2019, 15, 3432.

[28] S. Lanzalaco, L. J. Del Valle, P. Turon, C. Weis, F. Estrany, C. Alemán, E. Armelin, J. Mater. Chem. B 2020, 8, 1049.

[29] W. J. Zheng, N. An, J. H. Yang, J. Zhou, Y. M. Chen, ACS Appl. Mater. Interfaces 2015, 7, 1758.

[30] Y. Tan, D. Wang, H. Xu, Y. Yang, X. L. Wang, F. Tian, P. Xu, W. An, X. Zhao, S. Xu, ACS Appl. Mater. Interfaces 2018, 10, 40125.

[31] J. C. Breger, C. Yoon, R. Xiao, H. R. Kwag, M. O. Wang, J. P. Fisher, T. D. Nguyen, D. H. Gracias, ACS Appl. Mater. Interfaces 2015, 7, 3398.

[32] I. Apsite, G. Stoychev, W. Zhang, D. Jehnichen, J. Xie, L. Ionov, Biomacromolecules 2017, 18, 3178.

[33] Y. Yamamoto, K. Kanao, T. Arie, S. Akita, K. Takei, ACS Appl. Mater. Interfaces 2015, 7, 11002-11006.

[34] C. Yao, Z. Liu, C. Yang, W. Wang, X. Ju, R. Xie, L. Chu, Adv. Funct. Mater. 2015, 2980.

[35] W. S. Cobb, J. M. Burns, K. W. Kercher, B. D. Matthews, H. J. Norton, D. Ph, B. T. Heniford, J. Surg. Res. 2005, 129, 231.

[36] K. Junge, U. Klinge, A. Prescher, P. Giboni, M. Niewiera, V. S. M, Hernia 2001, 5, 
113.

[37] Y. J. Hwang, J. S. An, M. G. Mccord, S. W. Park, B. C. Kang, Fibers Polym. 2003, 4, 145.

[38] A. Mourran, H. Zhang, R. Vinokur, M. Möller, Adv. Mater. 2017, 29, 1604825.

[39] H. Banerjee, M. Suhail, H. Ren, Biomimetics 2018, 3, 15.

[40] M. A. C. Stuart, W. T. S. Huck, J. Genzer, M. Müller, C. Ober, M. Stamm, G. B. Sukhorukov, I. Szleifer, V. V Tsukruk, M. Urban, F. Winnik, S. Zauscher, I. Luzinov, S. Minko, Nat. Mater. 2010, 9, 101.

[41] Y. Ma, Y. Zhang, B. Wu, W. Sun, Z. Li, J. Sun, Angew. Chemie Int. Ed. 2011, 50, 6254.

[42] J. Kim, J. A. Hanna, R. C. Hayward, C. D. Santangelo, Soft Matter 2012, 8, 2375.

[43] H. Arazoe, D. Miyajima, K. Akaike, F. Araoka, E. Sato, T. Hikima, M. Kawamoto, T. Aida, Nat. Mater. 2016, 15, 1084.

[44] Z. J. Wang, C. Y. Li, X. Y. Zhao, Z. L. Wu, Q. Zheng, J. Mater. Chem. B 2019, 7, 1674.

[45] M. Hippler, E. Blasco, J. Qu, M. Tanaka, C. Barner-Kowollik, M. Wegener, M. Bastmeyer, Nat. Commun. 2019, 10, 1.

[46] ANNUAL BOOK OF ASTM STANDARDS VOLUME 0702, Textiles (II); Materials, A. S. for T. \&, Ed.; 2002. 


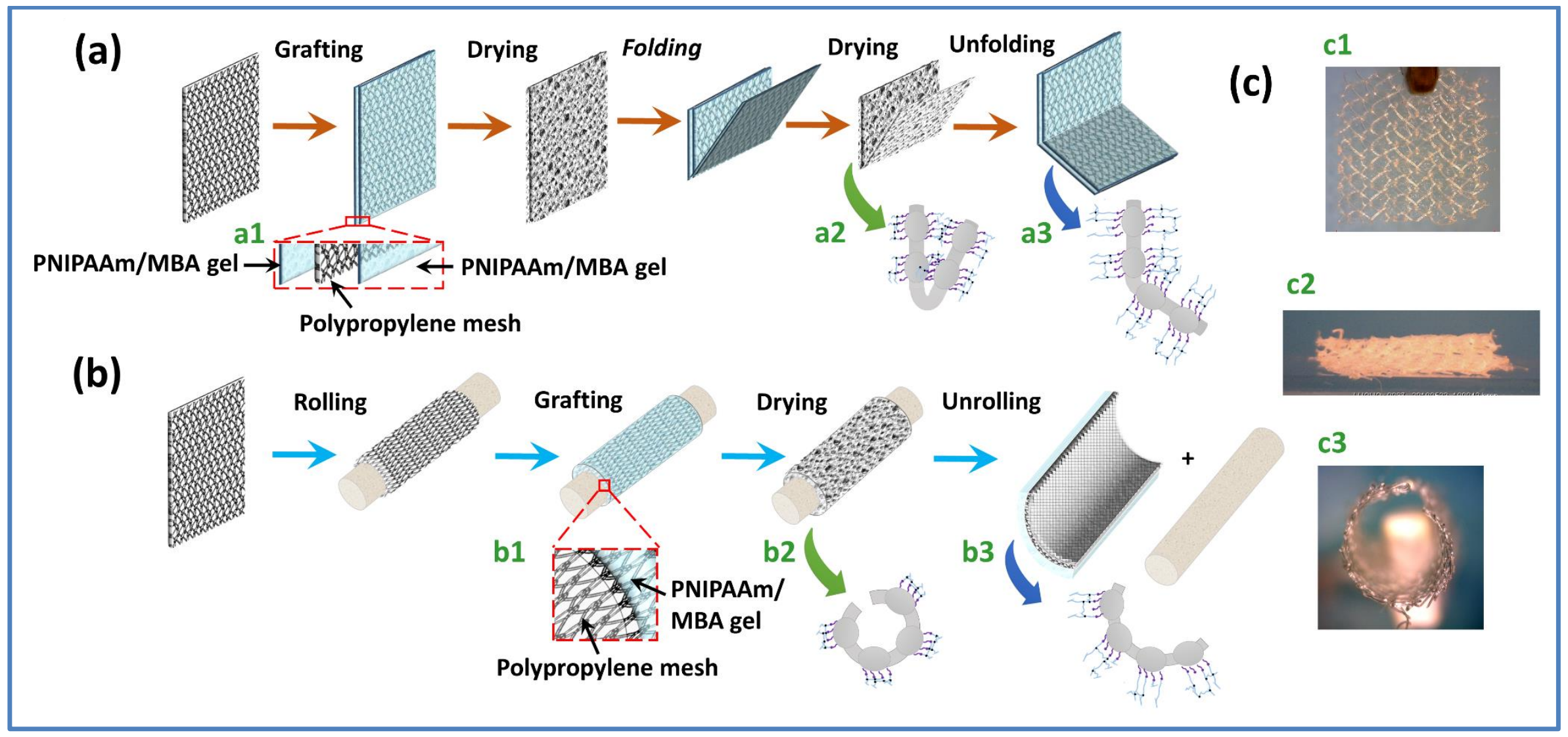

Figure 1. Scheme of the preparation of the smart materials: (a) bilayer and (b) monolayer iPP- $g$-PNIPAAm meshes. (a1-b1) Magnification of the PNIPAAm gel distribution onto the polypropylene mesh; (a2-b2), iPP- $g$-PNIPAAm meshes in its contracted shape after drying at $37{ }^{\circ} \mathrm{C}$; (a3-b3) iPP-g-PNIPAAm meshes in its expanded and unfolded/unrolled shapes. (c) Digital photographs of wet (c1) iPP-g-PNIPAAm mesh grafted during $2 \mathrm{~h}$ and dried at $37^{\circ} \mathrm{C}$ in its folded (c2) and rolled (c3) conformations. 
(a)
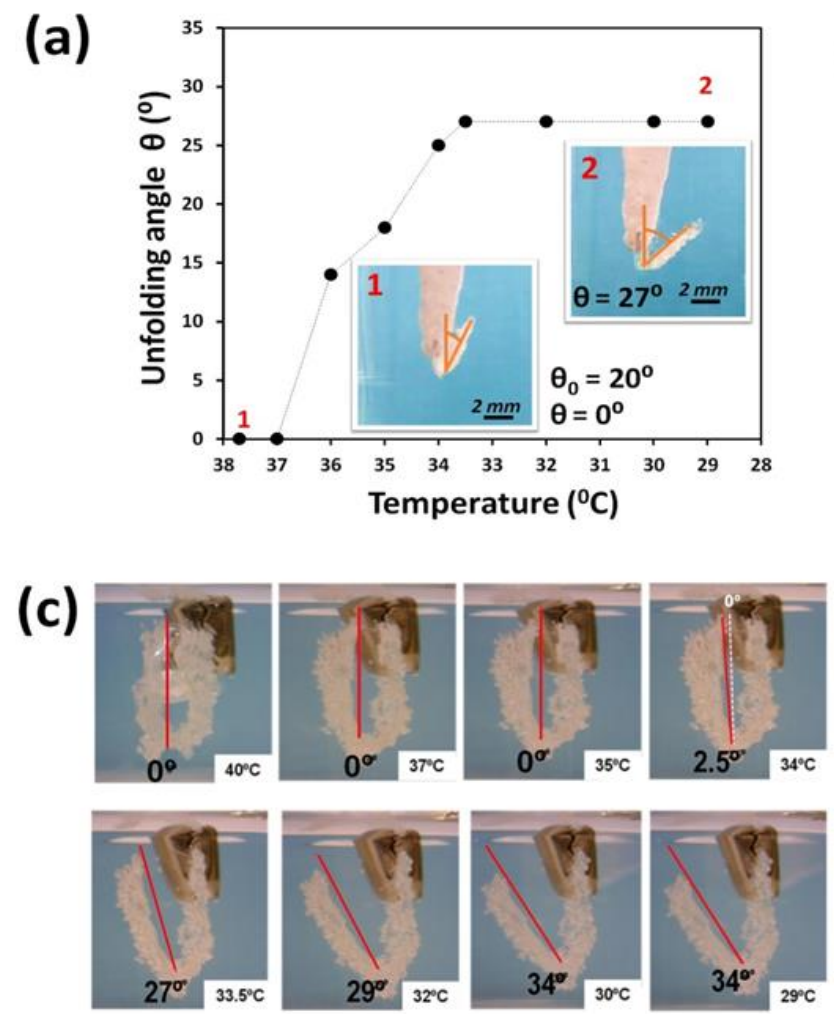

(b)

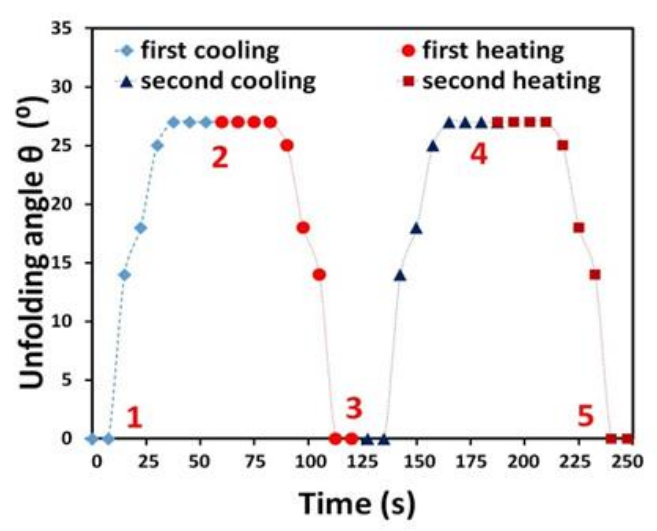

(d)

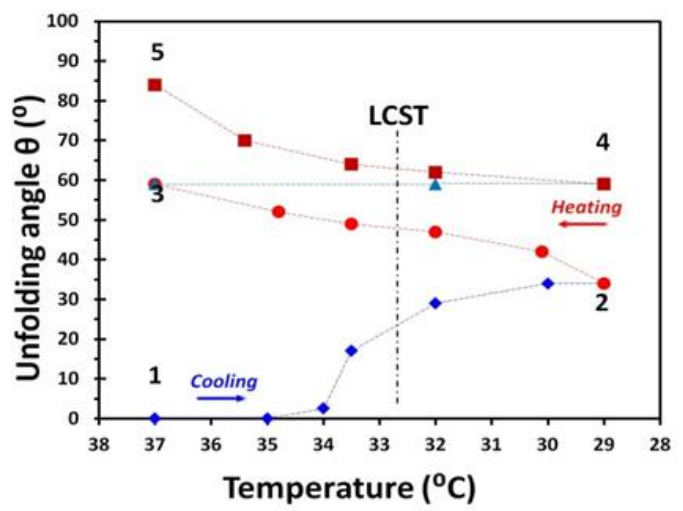

Figure 2. (a) Variation of unfolding angles versus cooling temperature for the bilayer iPP- $g$ PNIPAAm mesh prepared with $1 \mathrm{~h}$ of grafting reaction (the inset photographs represent the maximum folded and unfolded angles, $\theta=0$ and $27^{\circ}$, respectively). (b) Variation of unfolding angles versus time under cooling and heating cyclic experiments for iPP- $g$-PNIPAAm samples obtained at a grafting time of $1 \mathrm{~h}$ (i.e. very low content of PNIPAAm gel surrounding the PP fibres). The reversible behaviour of such tough-soft devices is evidenced. All data are related to the experiments in wet conditions, i.e. with the platforms immersed in the physiological solution of PBS. (c) Sequence of digital photographs showing the unfolding aperture (between $\theta=0^{0}$ and $\theta=34^{\circ}$ ) under cooling process (from $40^{\circ} \mathrm{C}$ to $29^{\circ} \mathrm{C}$ ) of a bilayer iPP- $g$-PNIPAAm platform prepared with $2 \mathrm{~h}$ of grafting reaction. (d) Variation of unfolding angles versus time under cooling and heating cyclic experiments for iPP-g-PNIPAAm samples obtained at a grafting time of $2 \mathrm{~h}$ (LCST: lower critical solution temperature). 


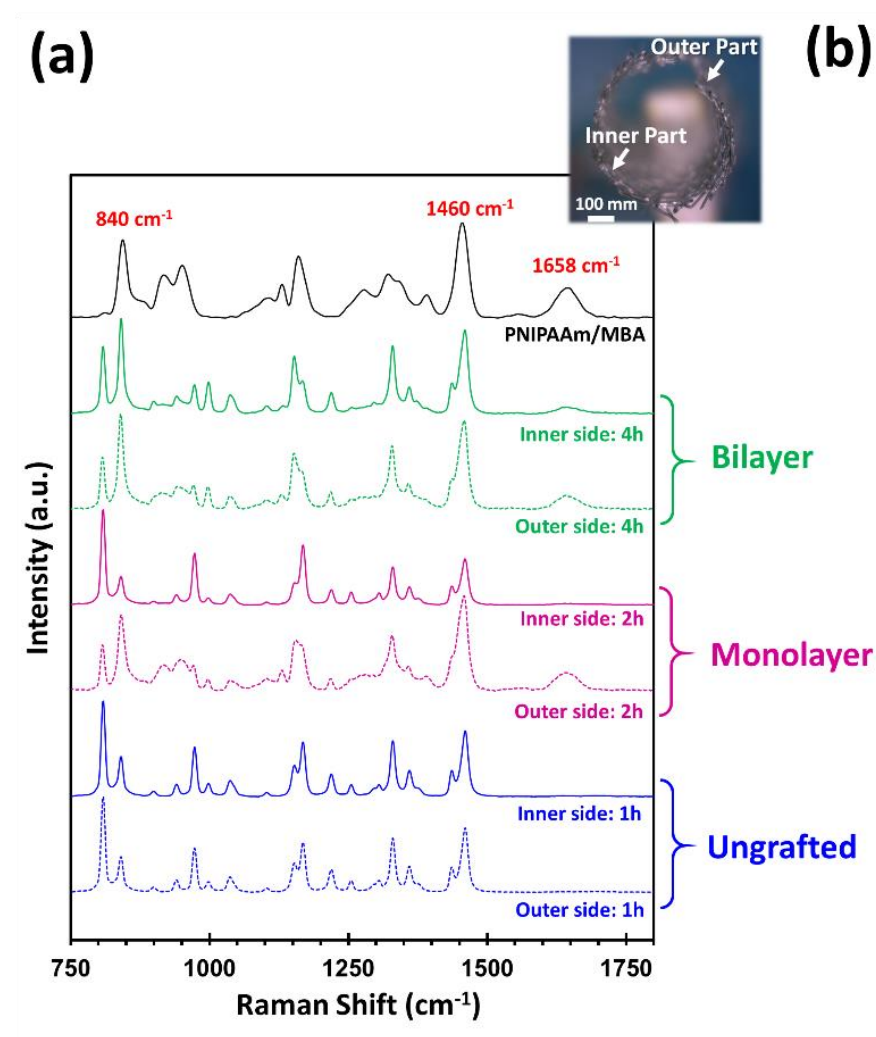

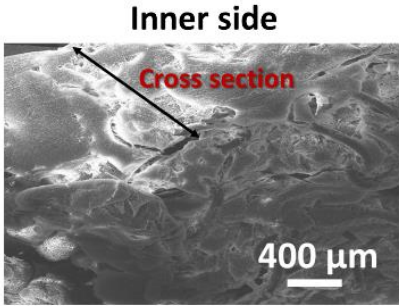

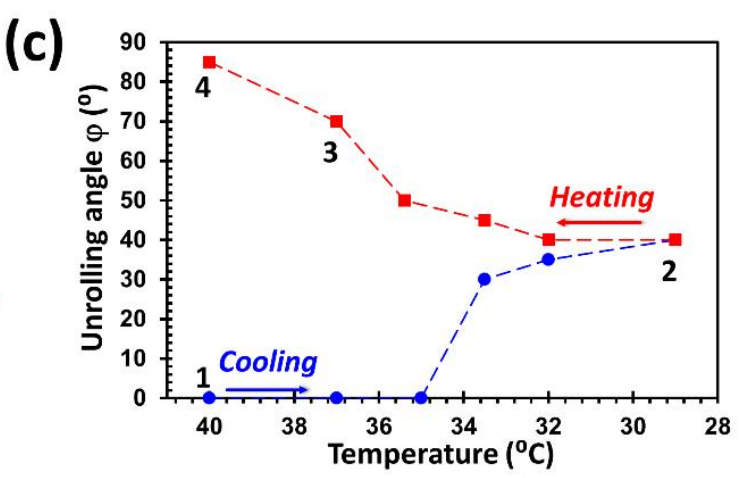

(e)

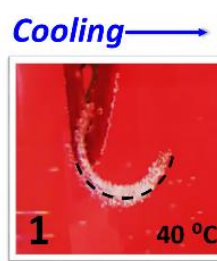

Outer side

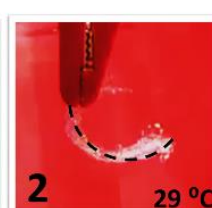

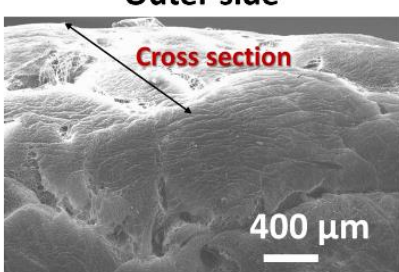

(d)

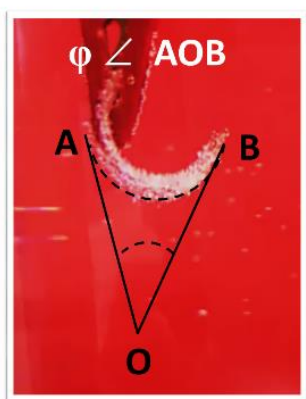

Heating $\longrightarrow$

Gel Morphology
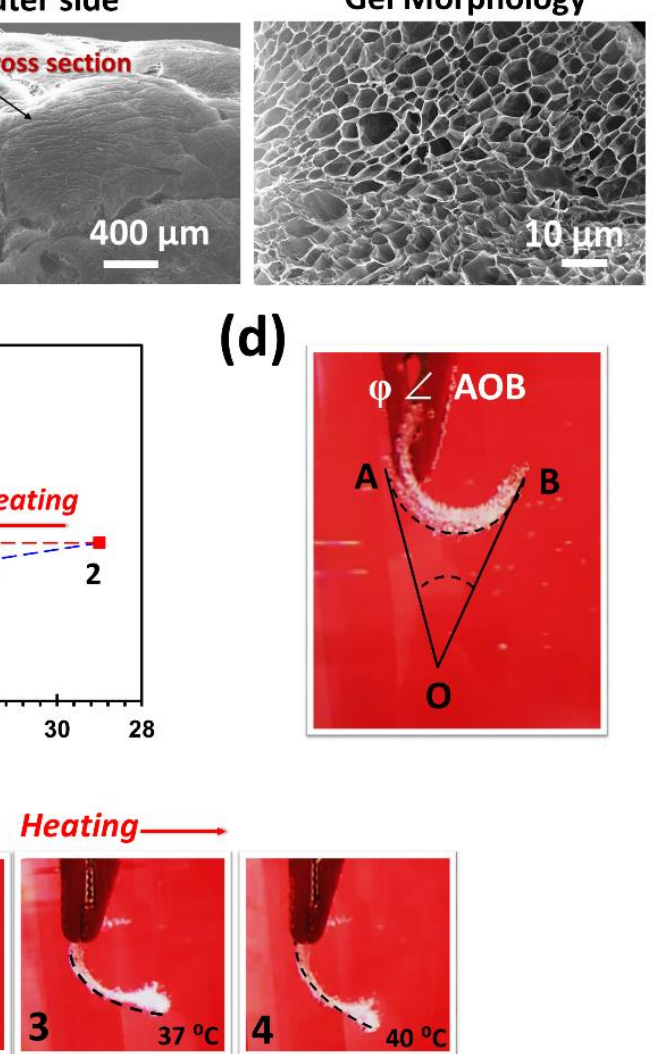

Figure 3. (a) Raman spectra for PP-g-PNIPAAm platforms in the rolled configuration. Spectra show the changes in the chemical structure of both the inner and the outer sides, after extraction of glass bare used to block the whole system and after purification (inset: photograph of the mesh in the fully rolled configuration). (b) SEM micrographs from cross-section of PP-g-PNIPAAm mesh (left and middle images) and morphology of the gel distribution (on right). (c) Unrolling angles variation versus cooling and heating temperatures for PP-g-PNIPAAm sample obtained at a grafting time of 2 h. (d) Scheme of measurement of the unrolling angle $(\varphi)$, (e) Digital photographs illustrating the mesh movement in cooling and heating steps at different temperatures (movie MV3 in ESI). 

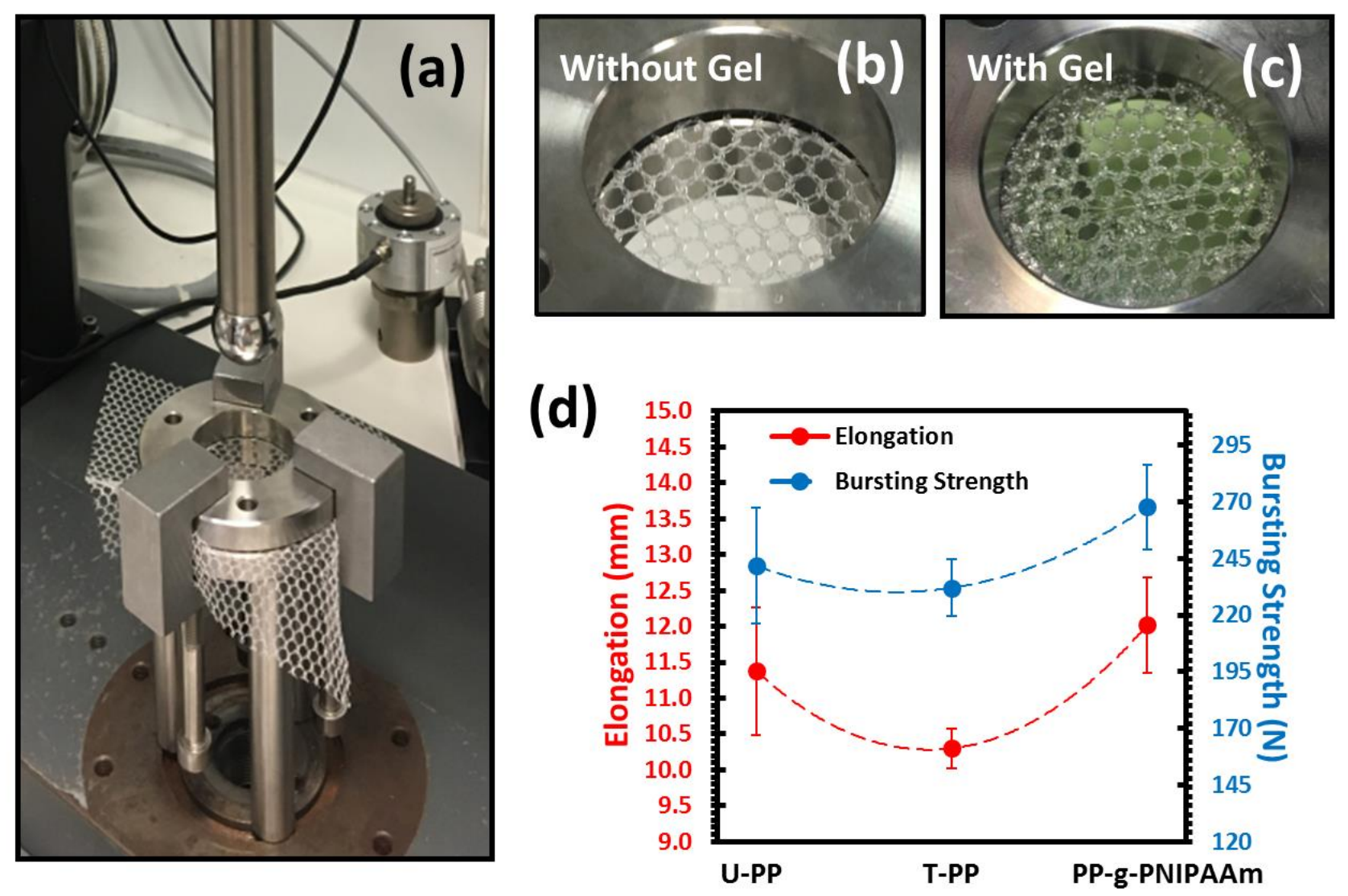

Figure 4. (a) Experiment set-up for bursting strength test of PP meshes; (b and c) images showing a detail of the meshes, without and with PNIPAAm gel, respectively, fixed to the support (before analysis); and (c) elongation and bursting strength versus mesh material: pristine PP mesh (U-PP), plasma-treated PP mesh (T-PP) and PP-g-PNIPAAm mesh. 


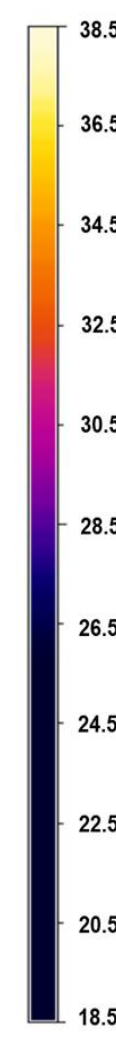

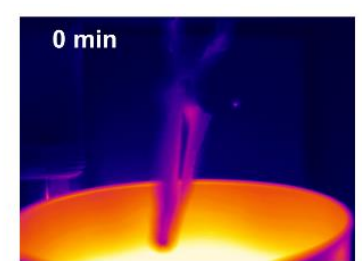
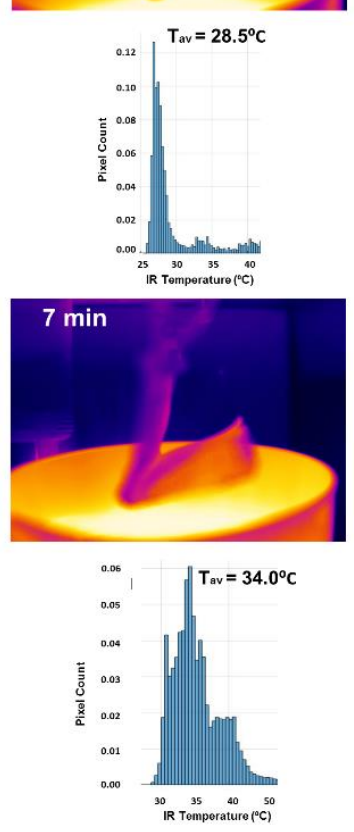
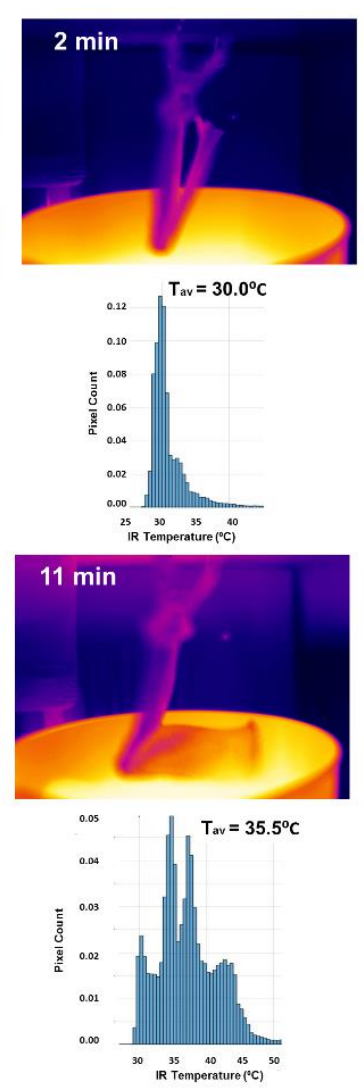
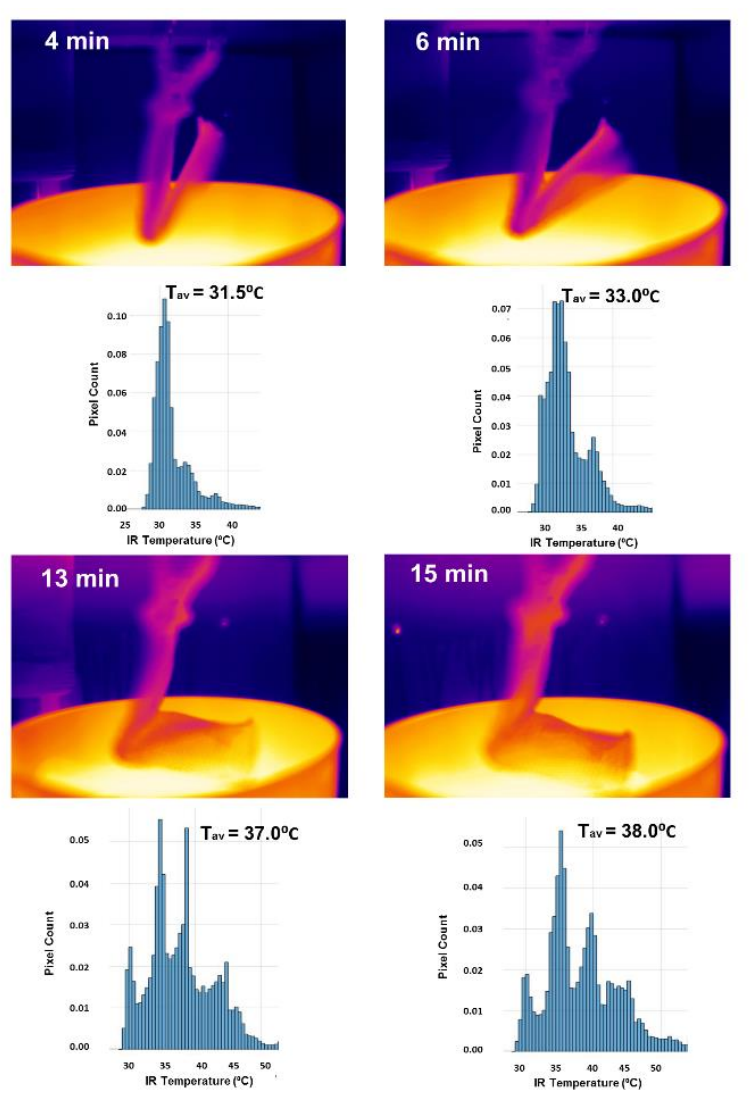

Figure 5. Infrared (IR) images from PP-g-PNIPAAm bilayer platforms after $2 \mathrm{~h}$ of graft reaction, at different temperatures, and the respective histograms obtained from image processing by using ThermaCAM Researcher ${ }^{\circledR}$ and Matlab $2017 b^{\circledR}$ softwares. The colourful bar (on left) shows the temperature distribution. Purple colour shows the lower temperature $\left(28.5^{\circ} \mathrm{C}\right)$, red colour shows higher temperature $\left(32.5^{\circ} \mathrm{C}\right)$, while the blue, as a background colour, is representative of the coldest temperature $\left(26.5^{\circ} \mathrm{C}\right)$. 


\section{TOC text and image}

The fourth dimension (4D) concept for creating dynamic devices, that can change their shape and/or function under controlled stimulus, is an emerging technology. Here, a smart responsive mesh material, able to self-evolve under temperature and humidity variations, has been developed. The specific stimulus response was assessed by thermal infrared imaging camera and optical microscope, for the macro- and microstructure evaluations, respectively.

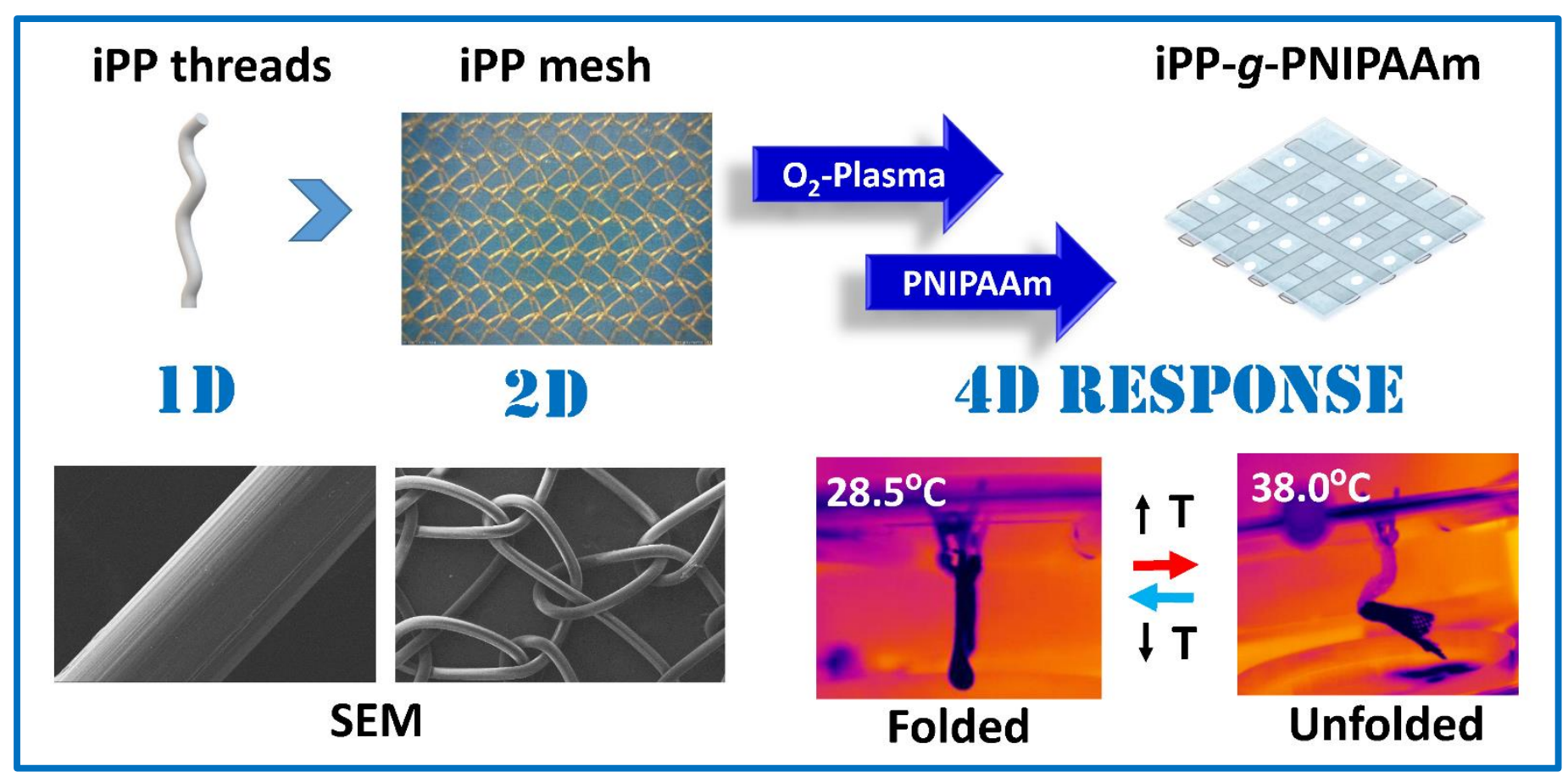

